\title{
Smart Network Price Policy for ISP Based on Traffic Prediction
}

\author{
Tingya Su \\ Portledge School, New York, USA \\ Email: tingyasu2001@gmail.com
}

How to cite this paper: Su, T.Y. (2021) Smart Network Price Policy for ISP Based on Traffic Prediction. Journal of Mathematical Finance, 11, 1-14.

https://doi.org/10.4236/jmf.2021.111001

Received: September 24, 2020

Accepted: February 1, 2021

Published: February 4, 2021

Copyright $\odot 2021$ by author(s) and Scientific Research Publishing Inc. This work is licensed under the Creative Commons Attribution International License (CC BY 4.0).

http://creativecommons.org/licenses/by/4.0/

\begin{abstract}
The explosion of traffic brings the challenges for Internet Service Providers (ISPs) to make a profit with the high cost of infrastructure and increased competition. This calls for economic mechanisms that can enable providers to allocate on-demand resources through the prediction of traffic volumes and adjust the price. In this paper, we analyze the network traffic pattern of mobile data and make an accurate prediction of traffic volumes through ARIMA and LSTM. Based on the analysis, we then suggest a scalable price strategy for ISPs to satisfy the various requirements of customers.
\end{abstract}

\section{Keywords}

Time-Series Analysis, Network Traffic Prediction, Telecommunication Traffic, ARIMA, LSTM, Deep Learning, ISP Pricing

\section{Introduction}

According to Cisco annual internet report, mobile users will increase to 5.7 billion, mobile connections will increase to 13.1 billion, and mobile traffic volume is estimated to reach almost one zeta-byte by 2023. Particularly, data traffic produced by smartphones accounts for 86 percent of all mobile data traffic as emergence of various mobile applications such as online chatting, mobile games, and online shopping [1]. The traffic explosion is pushing forward the development of fifth-generation (5G) networks, which is predicted to account for more than $10 \%$ of total mobile traffic by 2022 . Internet service providers (ISP) are facing a new challenge of maximizing profit through developing 5G networks and charging for customers. The $5 \mathrm{G}$ network market is two-sided market with a supply-demand balance depending on the network deployment cost and service requests. The price strategy published by ISP has a large impact on the potential demand for the service requested by customers, i.e., customers may not choose 
$5 \mathrm{G}$ networks if the price is too high. On the other hand, ISP cannot make a profit if the price is too low or the market-sharing is low. Thus, in order to make a profit, ISP needs to set a proper price strategy to attract more customers. Meanwhile, increasing the network utilization and providing on-demand service are also an effective strategy to reduce the cost, and then gain higher profit.

Previous literature has explored pricing strategy and resource allocation problems in networking services. Low and Lapsley proposed a convergence algorithm to flow control and maximize the aggregate source utility based on the transmission rates [2]. Marbach presented a pricing scheme where different users can receive data, which is different to address the social welfare maximization problem [3]. Heikkinen discussed the issue of optimal quality of service and the optimal linear pricing mechanism in the multi-service network [4]. However, these studies mainly focus on the maximization of social welfare and the case of small demand. They have not considered the customers' own interests and usage patterns and ignored the actual value of consumed network resources. These studies also overlooked the internet service provider's goal of profiting by providing network services. They pay less attention to the revenue maximization problem for network service providers. In this paper, we analyze the usage pattern of customers and predict the request volumes based on the historical data. We then design smart pricing schemes with temporal dynamics to improve ISPs' profit. ISPs can promote this novel service price strategy to balance resource utilization, optimize user experience, and attract new customers with customer-made packages based on the prediction of traffic volumes.

Moreover, future ISP prefers to provide on-demand services for customers for profit consideration. To improve the efficiency of network resources and deliver better user experience, the accurate traffic prediction model is required for ISP to allocate "just-right" amount of resources. Moreover, the network traffic prediction model would have numerous practical applications, including maintaining a stable network, optimizing user experience, and ensuring network security.

To address the aforementioned challenges, we first analyze the usage pattern of customers with the time series model and then predict the traffic volume through Auto-regressive Integrated Moving Average (ARIMA) model and Long Short-Term Memory neural networks (LSTM). Based on the analysis, we suggest the price strategy for ISPs.

Our contributions in this paper are summarized as:

- We analyze the traffic pattern of mobile data.

- We develop an Arima-based traffic prediction model.

- We develop an LSTM-based traffic prediction model.

- We design a smart pricing strategy for ISPs to promote a temporal dynamic service package to attract more users and then make a higher profit.

The rest of the paper is organized as follows. In Section 2, we review the previous works on network traffic prediction and ISP pricing policy. In Section 3, we discuss the problem design of our study. In Section 4, we introduce the traditional statistical-based algorithm and machine learning-based algorithm for the 
network traffic prediction. In Section 5, we evaluate and compare the performance of the prediction models. In Section 6, we discuss the ISP pricing strategy based on the network traffic prediction model we proposed. The final Section 7 concludes the study.

\section{Related Work}

Various past studies have examined the accuracy and effectiveness of numerous network traffic prediction models. The previous works can generally be divided into two categories, the statistical-based method and machine learning-based method. Iqbal has classified the previous network traffic prediction models into three categories, which are "classic time series-based predictors, Artificial Neural Networks-based predictors, and wavelet transform-based predictors" [5]. Boutaba examined the existing time series forecasting models for traffic prediction and classified them into "statistical analysis models and supervised ML models" [6]. Ramakrishnan demonstrated the effectiveness of RNN architecture to model network traffic data with a high degree of complexity and non-linearity [7]. Chen verified the high accuracy of the combination model for predicting nonlinear time-series data [8]. Nie proposed a prediction model called DBNG with a combination of Deep Belief Network and the Gaussian model [9]. This study will verify the most effective and accurate network traffic model based on previous studies.

The resource allocation optimization problem and the pricing policy of network services have also been discussed in many past studies. Multiple previous researches aimed to optimally allocate the available resources for providing better network quality-of-service (QoS). Ahmed presented an integrated algorithm with the optimal traffic-dependent allocation rate for both high-traffic and low-traffic situations [10]. The proposed pricing policy of this study can effectively help the network providers to flatten traffic load and decrease the cost for the users. Harks proposed a utility proportional fair resource allocation model for users with both elastic and real-time traffic [11]. Tychogiorgos also proposed the utility proportional fair algorithm for the optimization of rate allocation in high-SINR wireless network [12]. The approach showed a more efficient and effective performance than the traditional bandwidth proportional fair approach. Based on the prior studies, we will propose an algorithm for fair resource allocation and pricing schemes for future $5 \mathrm{G}$ networks.

\section{Traffic Prediction Models}

In this section, we first formulate traffic prediction problems by mathematical notations. Then, we use the time series approach ARIMA and deep learning approach LSTM to construct the prediction model.

\subsection{Problem Formulation}

Denote $y_{t}$ as the traffic volume at time period $t$. The historic traffic data 
fromtime $t-k$ to time $t$ can be represented as $Y=\left\{y_{t-i}, \cdots, y_{t}\right\}$. Our objective is to predict the future traffic volume from time $t+l$ to time $t+l$. Denote $\hat{y}_{t+1}$ as the predicted traffic volume at time $t+l$ while $y_{t+1}$ as the ground truth traffic. The problem can be formulated as:

$$
\begin{aligned}
& \min \sum_{i=t+1}^{t+l}\left\|\hat{y}_{i}-y_{i}\right\|^{2} \\
& \hat{y}_{t+1}=f\left(y_{t-i}, \cdots, y_{t}\right)
\end{aligned}
$$

\subsection{Time Series Analysis}

In this study, we will apply the Auto-regressive Integrated Moving Average (ARIMA) model, which is one of the most popular statistical analysis models for time series forecasting. ARIMA model is a combination of the Auto-regressive (AR) model and Moving average (MA) model. The AR model predicts future behavior based on past behavior according to the relation between a value in the present $\left(y_{t}\right)$ and previous value $\left(y_{t-k}\right)$. The auto-regression (AR) model is expressed as follows:

$$
y_{t}=\mu+\sum_{i=1}^{p} \gamma_{i} y_{t-i}+\epsilon_{t}
$$

where $\mu$ is a constant, $p$ is the order, $\gamma_{i}$ is the coefficient for the lagged variable in time $(t-p)$, and $\epsilon_{t}$ is the white noise at $t$.

The Moving Average (MA) model shows the possibility of relation between a present value $\left(y_{t}\right)$ and residuals from previous periods. The moving average (MA) model is expressed as follows:

$$
y_{t}=\mu+\epsilon_{t}+\sum_{i=1}^{q} \theta_{i} \epsilon_{t-i}
$$

where $\mu$ is a constant, $q$ is the order, $\theta_{i}$ is the coefficient for the lagged variable in time $(t-q)$, and $\epsilon_{t}$ is the random error at $t$.

The definition of ARIMA model is expressed as below as a combination of the AR and MA model:

$$
y_{t}=\mu+\sum_{i=1}^{p} \gamma_{i} y_{t-i}+\epsilon_{t}+\sum_{i=1}^{q} \theta_{i} \epsilon_{t-i}
$$

The ARIMA $(p, d, q)$ model combines the $p$ auto-regression terms and $q$ moving average terms. The $p$ is the order of auto-regression; $d$ is the degree of difference, and $q$ is the order of moving average.

\subsection{LSTM Model}

This paper applies the Long Short-Term Memory Neural Networks (LSTM) in network traffic prediction in addition to the ARIMA model. The LSTM neural network is ideal for our study due to its ability to capture sequence information and learn long term dependencies. LSTM is a special Recurrent Neural Network (RNN) with the memory cell's ability to automatically determine the optimal time lags for prediction. LSTM develops with short-term and long-term memory components to improve the traditional RNNs by solving the vanishing gradient problem [13]. The LSTM is proposed with the capability to train the time se- 
quence with long time lags. Thus, we will apply LSTM for our time series data. A typical LSTM unit is accomplished by the input gate it, the forget gate $f_{t}$, the cell state $c_{t}$, and output gate $\sigma_{t}$ at time $=t$ [14]. Let $\sigma$ be the standard sigmoid function, $W$ stands for the weight matrix, $b$ stands for the bias of the gates, and $\odot$ be the scalar product of two vectors or matrices [14], the functions and equations of the LSTM model components can be expressed as follows. The input gate reads and processes the extent of newly coming data:

$$
i_{t}=\sigma\left(W_{i}\left[h^{t-1}, x^{t}\right]+b^{i}\right)
$$

The cell state transfers and decides whether to keep or forget the relative information throughout the sequence processing.

$$
C_{t}=f_{t} \odot C_{t-1}+i_{t} \odot \overline{C_{t}}
$$

The forget gate decides the optimal time lags for the prediction of the input.

$$
f_{t}=\sigma\left(W^{f}\left[h^{t-1}, x^{t}\right]+b^{f}\right)
$$

The output gate generates the values in the cell and calculates the yielded value of the cell.

$$
o_{t}=\sigma\left(W^{o}\left[h^{t-1}, x^{t}\right]+b^{o}\right)
$$

with the function of these gates, the LSTM model can determine the arbitrary time lags with long dependency for our time-series datasets. Each sigmoid layer yields a value between zero and one to determine the amount of every segment of data for output.

\section{Evaluation}

\subsection{Dataset Description}

The dataset in this study consists of data collected from the Telecom Italia cellular network from the city of Milano, Italia. The data is obtained with a time interval of 600,000 milliseconds $(10 \mathrm{~min}$ ) over 62 days from 11/01/2013 to 01/01/2014. The short message service (SMS), call activity, and internet traffic activity is incorporated into the dataset. The spatial distribution of the cellular traffic dataset is gathered and represented in a grid with square cells. The area of Milan is composed of a grid overlay of 1000 squares, each with a size of about $235 \times 235$ meters [15]. In this paper, we focus on the traffic data of grid 0 . The traffic volumes of SMS, call, and internet are demonstrated in Figure 1.

\subsection{Time Series Analysis}

Before performing the forecast, we observed the characteristics of our time series dataset. Figure 1 shows the traffic volumes of SMS, call and Internet, which all have obvious daily patterns. To better learn the traffic pattern, we use additive decomposition method to extract the trend, seasonality, and residuals. As shown in Figure 2, the traffic volumes have a relatively stable trend over time. A local upward trend exhibits around 2013/12/22, which might potentially influence 


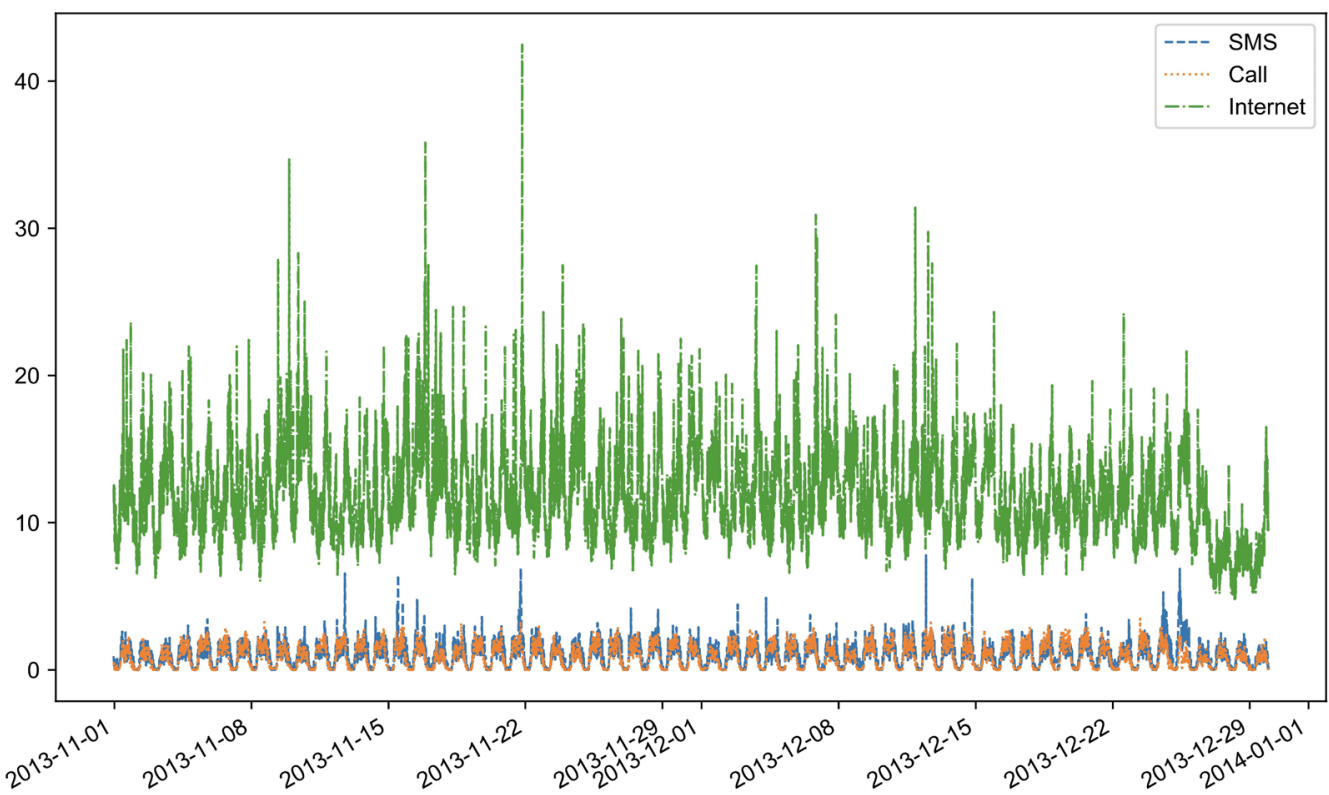

Figure 1. The traffic volume of SMS, call and internet.
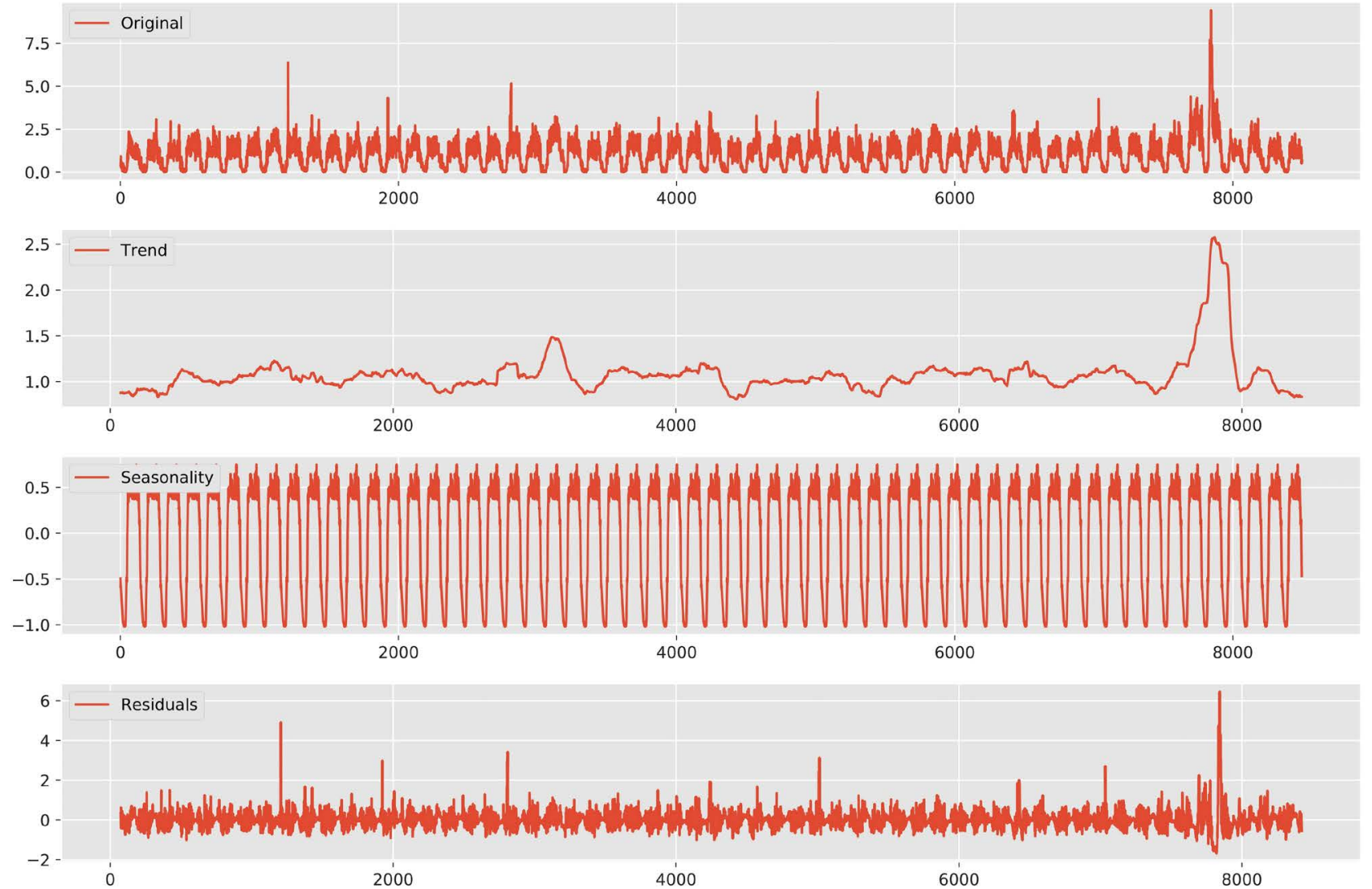

Figure 2. Decomposition of SMS data.

the forecast. We can see that SMS, call, and Internet traffic volumes all exhibit seasonal patterns over each day. The daily pattern is useful in forecasting and obtaining a good fit (Figure 3, Figure 4). 

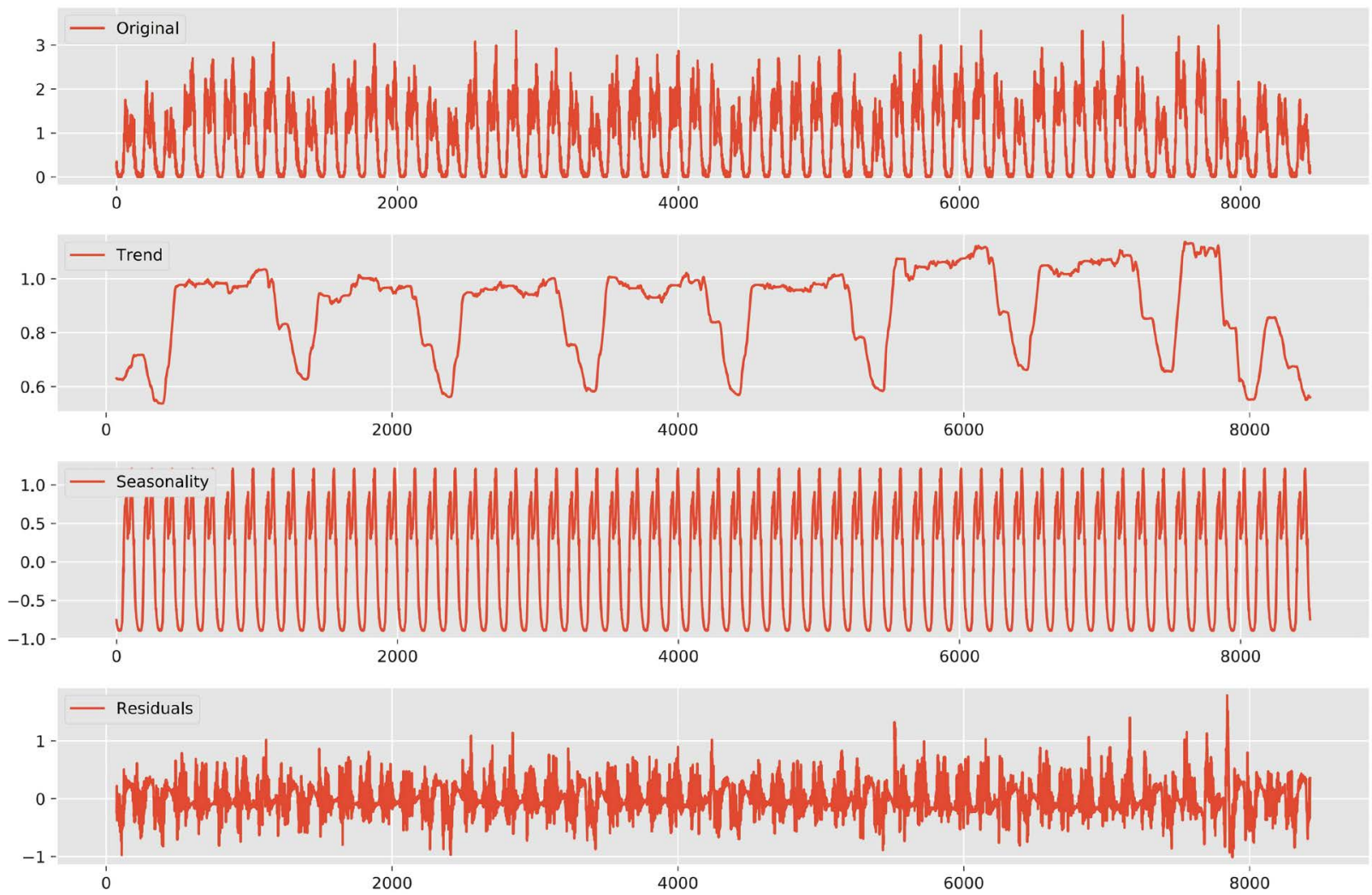

Figure 3. Decomposition of call data.
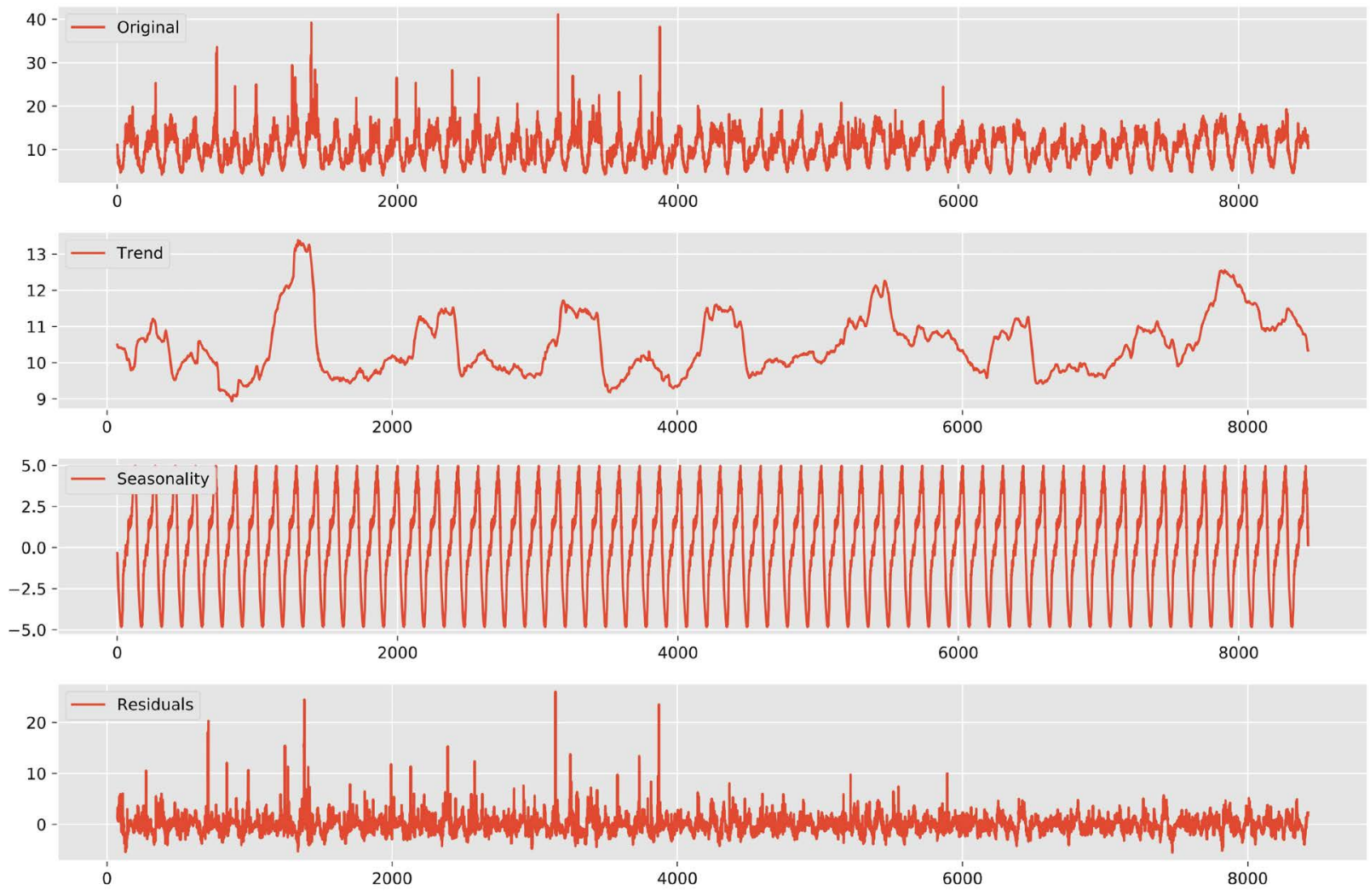

Figure 4. Decomposition of internet data. 


\subsection{Experimental Results of ARIMA Model}

We first work with the traditional statistical-model to predict our time series data. In determining the optimal parameters for our ARIMA model, this study uses a few estimators as reference. Akaike's Information Criterion (AIC) is helpful for estimating the orders $(p, d)$ of the ARIMA model. It can be written as:

$$
\mathrm{AIC}=-2 \log (L)+2(p+q+k+1)
$$

where $k$ is the number of estimated parameters in the model and $\mathrm{L}$ is the maximized maximum likelihood of the data. The Mean Squared Error (MSE) value, which measures the average squared difference between the estimated values and the actual value, can also be used to assess the adequacy of future values to our data. MSE is defined as follows:

$$
\operatorname{MSE}=\frac{1}{n} \sum_{i=1}^{n}\left(y_{i}-y_{i}\right)^{2}
$$

Lower AIC and MSE values indicate a better-fit model. Table 1 displays all the statistical results of different ARIMA parameters. The ARIMA $(1,0,1)$ is relatively the best model for the SMS, as it has the minimum AIC and MSE values. The ARIMA $(1,1,2)$ is chosen for the call traffic because of its lowest AIC and test MSE values. For the internet traffic, the $\operatorname{ARIMA}(2,1,0)$ is chosen due to its lowest MSE value compared to other parameters.

Then we present the performance of the selected ARIMA $(1,0,1)$ model on SMS traffic prediction in Figure 5. It shows a high-level accuracy of the predicted traffic against the actual SMS traffic. Figure 6 is the result of ARIMA (1, $1,2)$ for predicting call traffic. The performance of this selected model is considerably accurate as well, as the difference between the actual and predicted values is fairly small. Figure 7 reports the result of ARIMA $(2,1,0)$ for internet traffic prediction, which also shows a relatively accurate forecasting. Overall, these models all have high accuracy and can be used for simulate future Call Detail Records (CDRs) traffics.

Table 1. Statistical results of different ARIMA parameters.

\begin{tabular}{cccccccccc}
\hline \multicolumn{3}{c}{ SMS } & \multicolumn{9}{c}{ Call } \\
\hline$(p, d, q)$ & AIC & Test MSE & $(p, d, q)$ & AIC & Test MSE & $(p, d, q)$ & AIC & Test MSE \\
\hline$(1,0,0)$ & 8439.888 & 0.185 & $(1,0,0)$ & 632.066 & 0.064 & $(1,0,0)$ & $32,556.51$ & 1.236 \\
$(1,1,0)$ & 7749.470 & 0.167 & $(1,1,0)$ & -699.093 & 0.055 & $(1,1,0)$ & $31,738.12$ & 1.083 \\
$(0,1,0)$ & 9041.606 & 0.194 & $(0,1,0)$ & 859.627 & 0.065 & $(0,1,0)$ & $33,092.29$ & 1.224 \\
$(0,0,1)$ & 14646.12 & 0.444 & $(0,1,1)$ & -862.518 & 0.054 & $(0,1,1)$ & $31,186.21$ & 1.055 \\
$(1,0,1)$ & 6959.456 & 0.155 & $(0,2,1)$ & 871.574 & 0.065 & $(1,0,1)$ & $31,080.03$ & 1.050 \\
$(0,1,1)$ & 7069.868 & 0.156 & $(1,0,1)$ & -921.965 & 0.054 & $(1,1,2)$ & $31,177.21$ & 1.056 \\
$(2,0,0)$ & 7463.678 & 0.163 & $(1,1,2)$ & -1218.69 & 0.052 & $(2,0,0)$ & $31,491.65$ & 1.085 \\
$(2,1,0)$ & 7283.491 & 0.158 & $(2,0,0)$ & -793.891 & 0.055 & $(2,1,0)$ & $31,333.62$ & 1.030 \\
$(2,1,1)$ & 7039.183 & 0.156 & $(2,1,0)$ & -955.677 & 0.054 & $(2,1,1)$ & $31,178.38$ & 1.057 \\
\hline & & & & & & & &
\end{tabular}




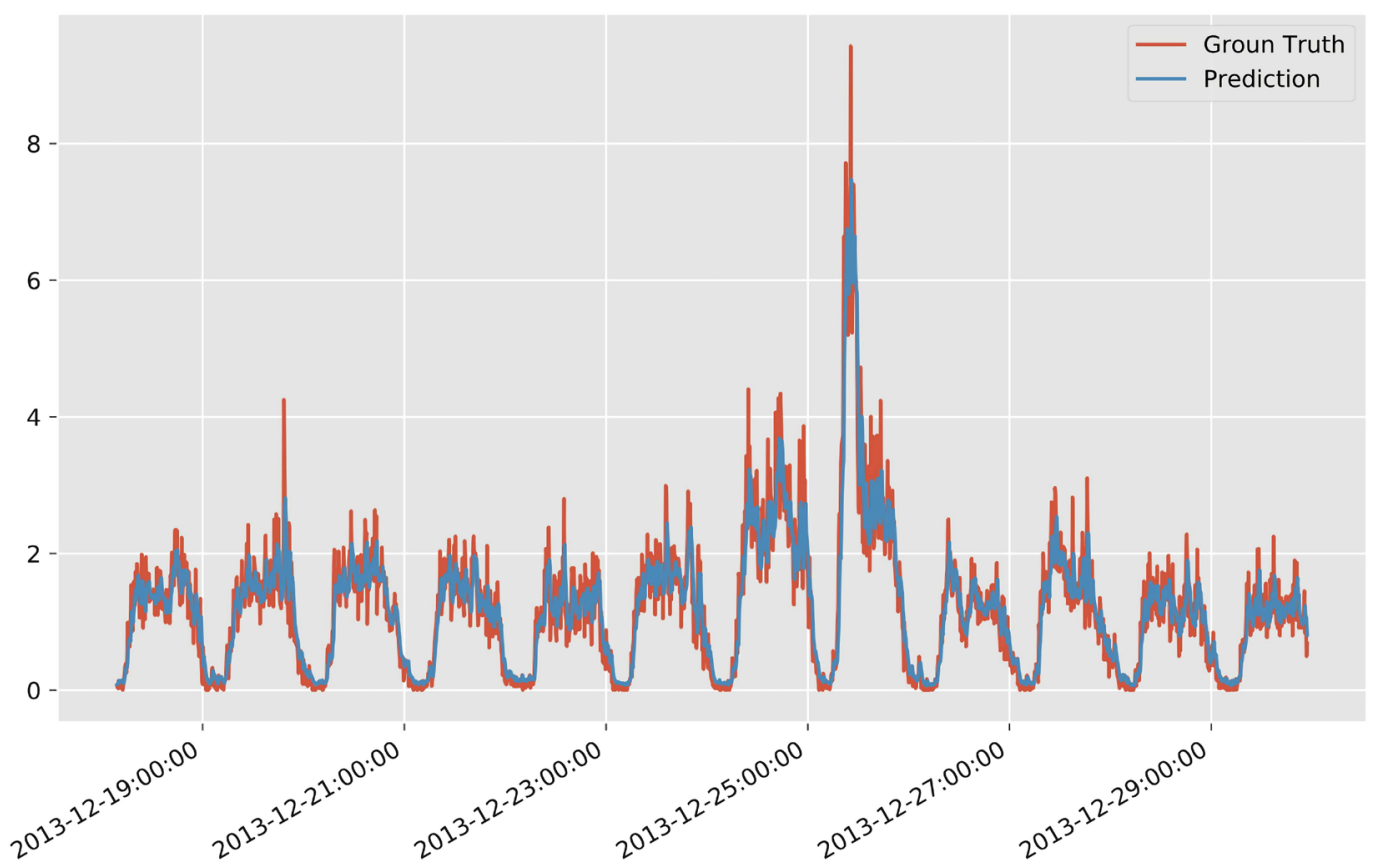

Figure 5. Plot for real value vs. predicted value for SMS using ARIMA.

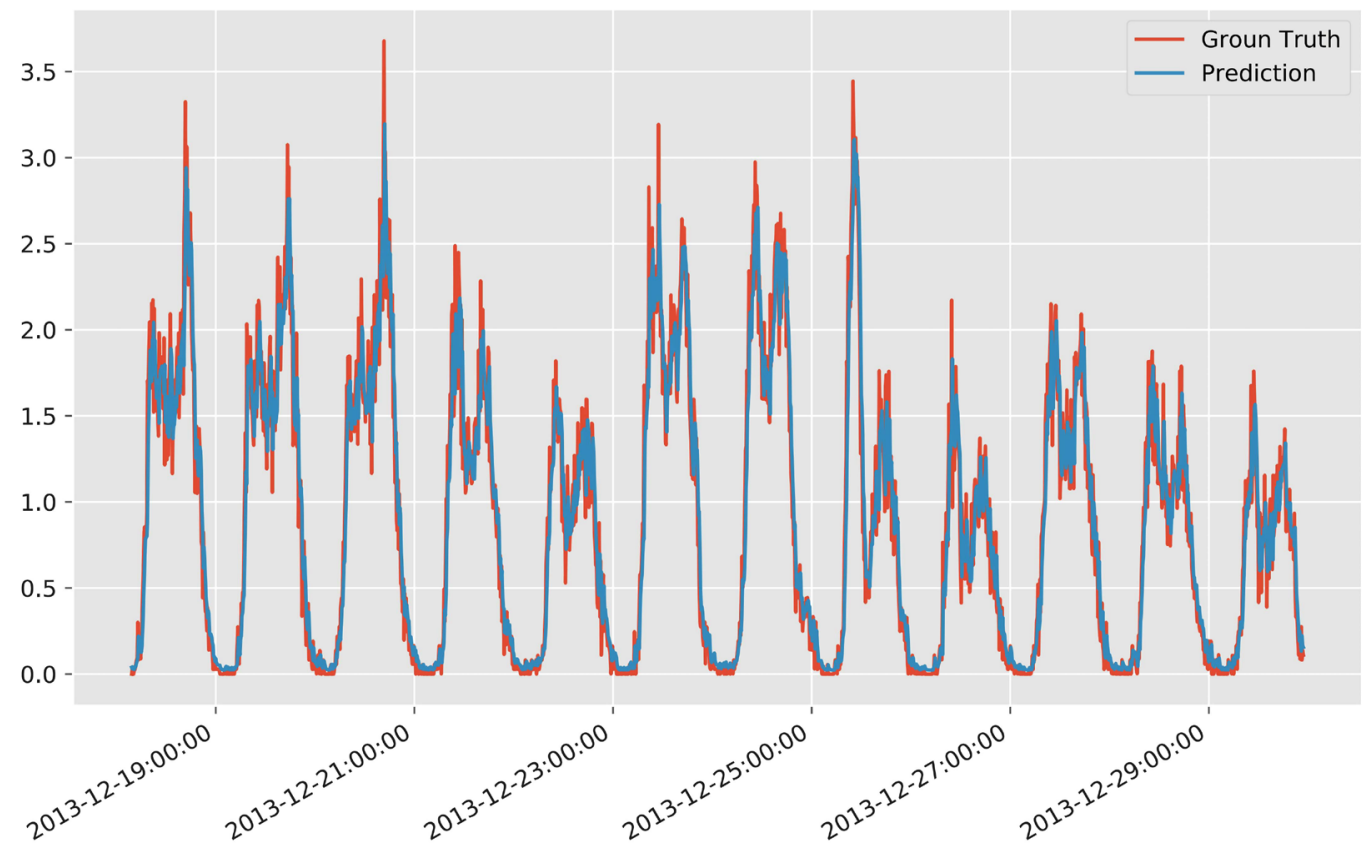

Figure 6. Plot for real value vs. predicted value for call using ARIMA.

\subsection{Experimental Results of LSTM Models}

The research uses the LSTM model in Keras which contains one LSTM layer with 128 nodes, and three Dense layers with 128, 256, 64 nodes respectively. The prediction results are shown in Figures 8-10. It is found that the LSTM is capable of predicting the traffic with a small difference between the actual and predicted value. 


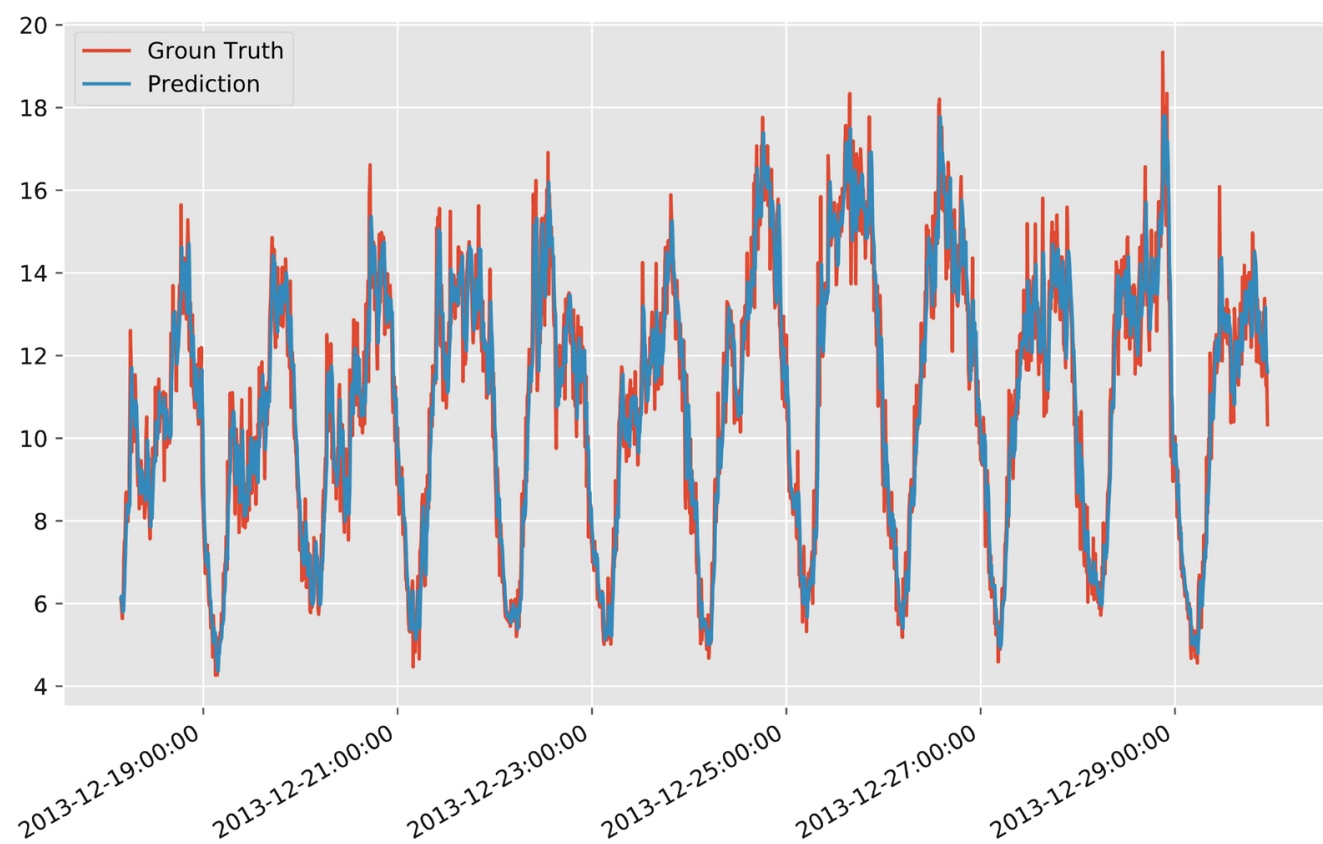

Figure 7. Plot for real value vs. predicted value for internet using ARIMA.

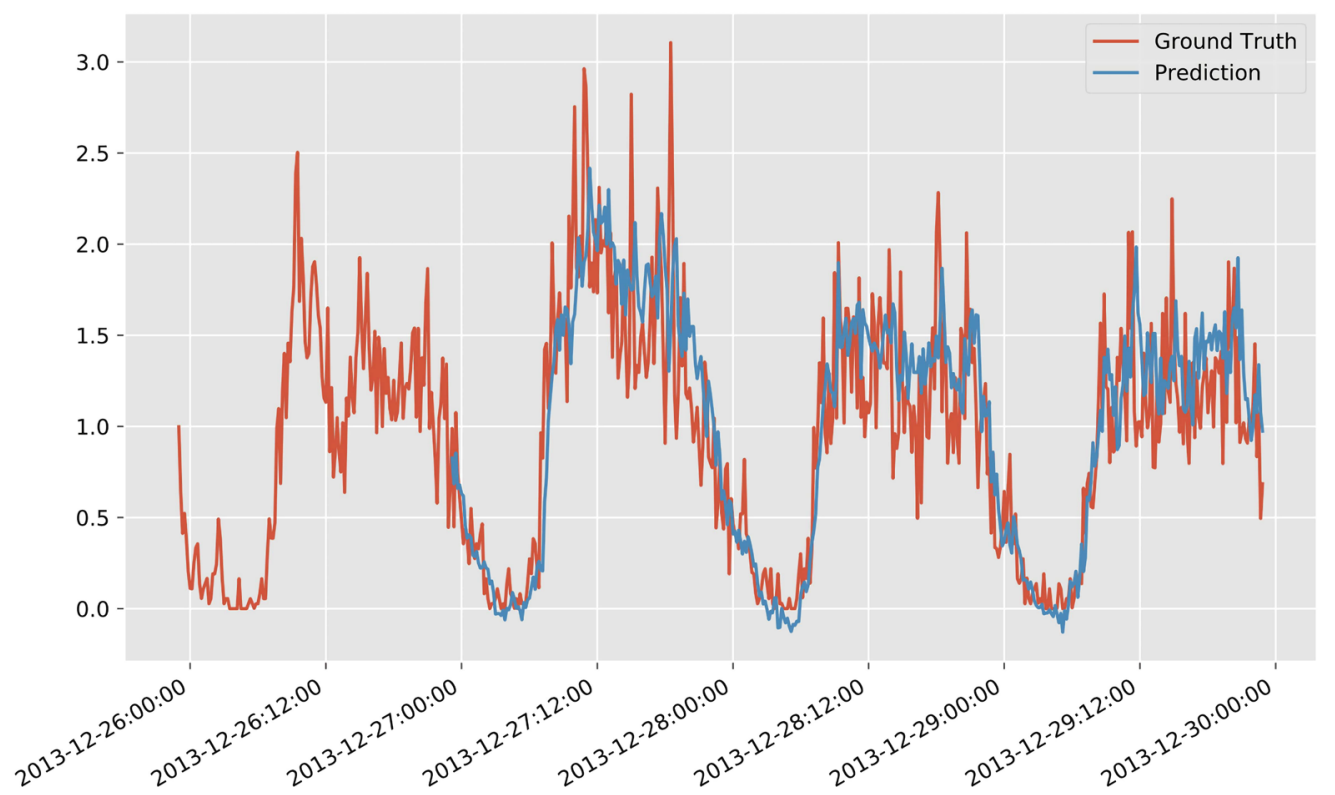

Figure 8. Plot for real value vs. predicted value for SMS using LSTM.

\subsection{Performance Evaluation of the Models}

In this section, we will evaluate the precision and accuracy of our ARIMA and LSTM models for predicting the network traffic datasets. We will compare the models with respect to their performance in reducing error rates. We use the assessment metric of the Root-Mean-Square Error (RMSE) to measure the accuracy of the prediction model. RMSE measures the square root of the average of the residuals between the actual and predicated values, which can be computed as follows: 


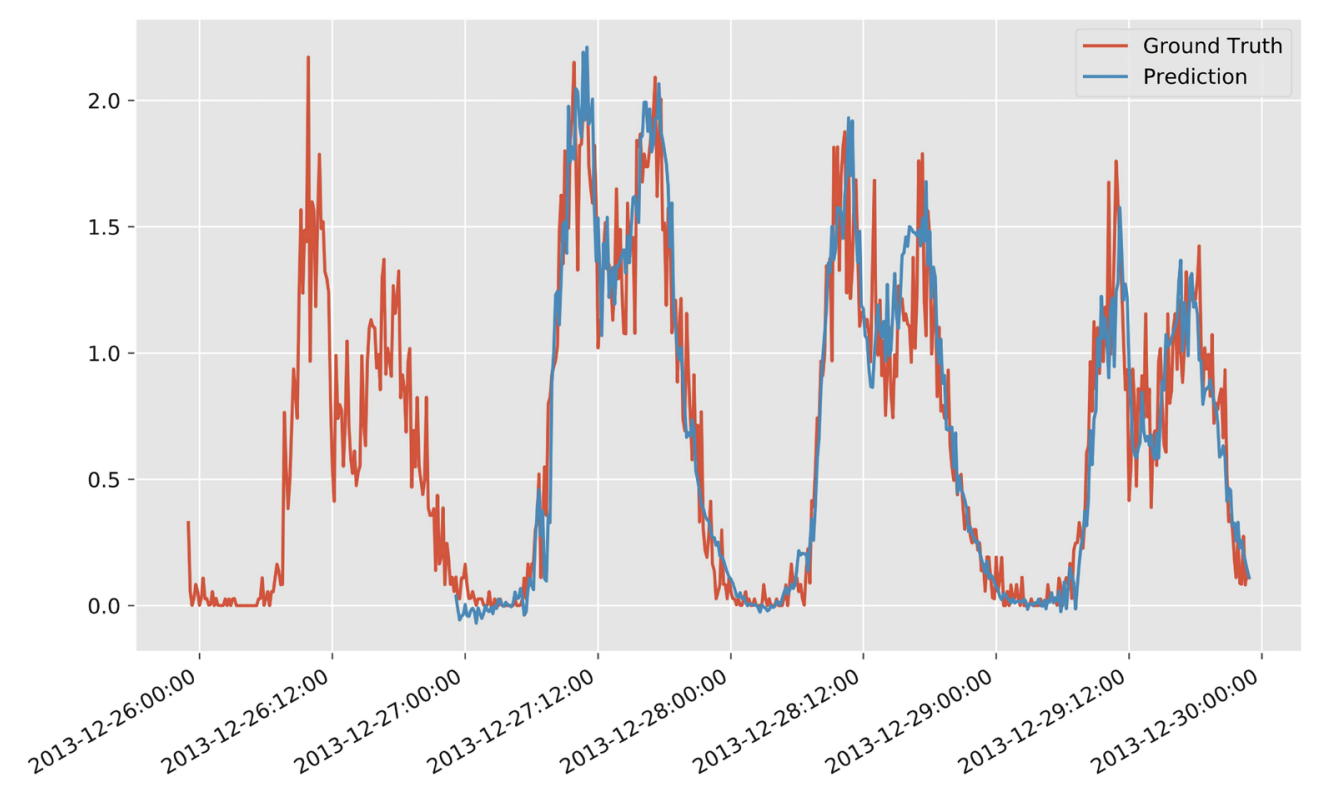

Figure 9. Plot for real value vs. predicted value for call using LSTM.

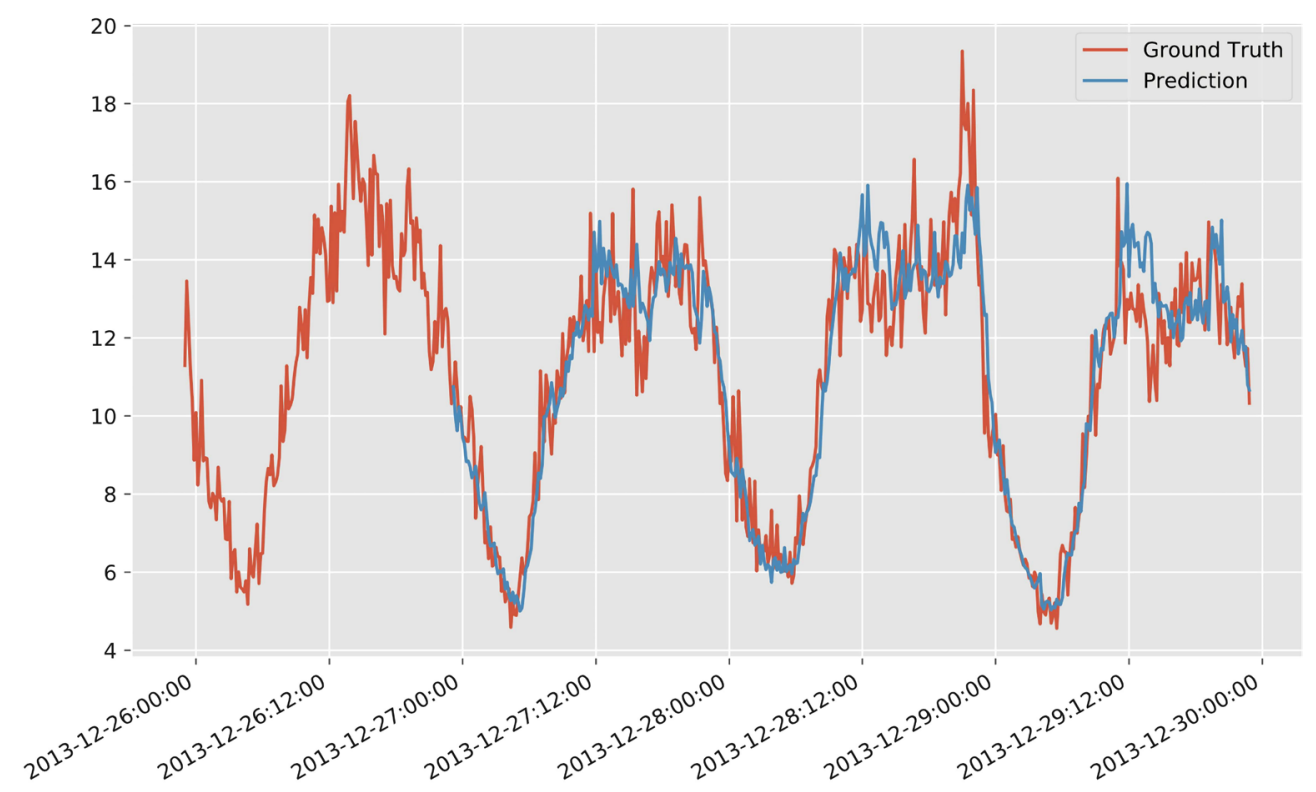

Figure 10. Plot for real value vs. predicted value for internet using LSTM.

$$
\mathrm{RMSE}=\sqrt{\frac{1}{N} \sum_{i=1}^{N}\left(x_{i}-\hat{x}_{i}\right)^{2}}
$$

In this equation, $N$ is the sample size; $x_{i}$ is the actual value; $\hat{x}_{i}$ is the predicted value. The smaller RMSE value indicates less error between the predicted and observed values. As shown in Table 2, the smaller RMSE values indicate that LSTM-based models outperform ARIMA-based models for SMS and call traffic prediction. For internet traffic, ARIMA is relatively more accurate with less error. Overall, the models all have considerably high level of accuracy and can be applied for future network traffic prediction. 
Table 2. The RMSEs of ARIMA and LSTM models.

\begin{tabular}{ccc}
\hline Models & ARIMA & LSTM \\
\hline SMS & 0.39 & 0.33 \\
Call & 0.23 & 0.19 \\
Internet & 1.02 & 1.05 \\
\hline
\end{tabular}

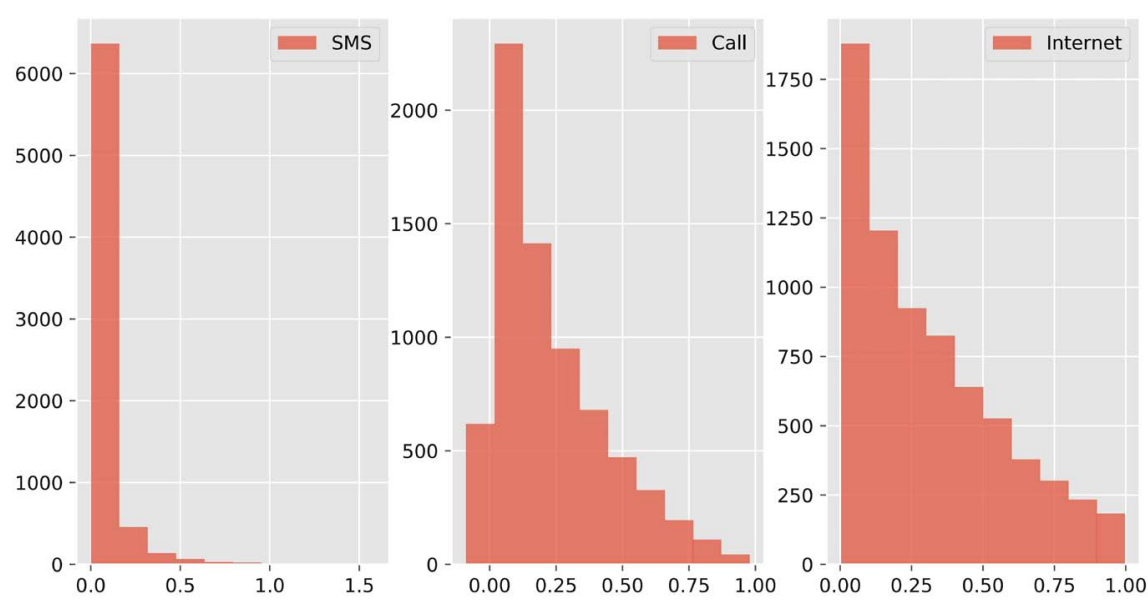

Figure 11. Hist of SMS, call, internet percentage in total cost.

\section{ISP Pricing Strategy}

Based on the prediction of traffic volumes, ISPs can allocate resources on-demand. Furthermore, ISPs can promote a "smart price" strategy to balance the traffic volumes in the temporal dimension. We have analyzed the percentage of SMS cost, call cost, and Internet cost in total cost respectively through the data set from Liantong ISP. From Figure 11, the call is still the most important application of smartphones while the SMS is much less used now due to the development of online chat applications. ISPs may consider promoting call and Internet packages for diverse requirements. Meanwhile, ISPs can promote the dynamic Internet price strategy which is negatively related to the traffic volume, i.e., cheap Internet price at midnight.

\section{Conclusion}

This paper analyzes the traffic volumes of SMS, call and Internet through time series analysis. Based on the traffic pattern, we use ARIMA and LSTM models to predict traffic and achieve high accuracy. This study compares and evaluates the performance of the models for the network traffic prediction. The research finds that the deep learning-based algorithm has relatively better performance than traditional statistical-based algorithm with a higher accuracy. Our developed model could be used by ISPs in improving network management and services. This study proposes the smart price strategy for ISP to better manage the on-demand resources and dynamicly adjust the price in different time of the day. Our smart pricing scheme could help ISP to increase their profit by balancing 
resource utilization and providing better services.

\section{Acknowledgements}

A special gratitude I give to my instructor, $\mathrm{Ms}$. $\mathrm{Xu}$, for her valuable and constructive suggestions during the planning and development of this research work. Her willingness to give her time so generously has been very much appreciated.

\section{Conflicts of Interest}

The author declares no conflicts of interest regarding the publication of this paper.

\section{References}

[1] Global Mobile Data Traffic Forecast (2019) Cisco Visual Networking Index: Global Mobile Data Traffic Forecast Update, 2017-2022.

[2] Low, S.H. and Lapsley, D.E. (1999) Optimization Flow Control. I. Basic Algorithm and Convergence. IEEE/ ACM Transactions on Networking, 7, 861-874.

https://doi.org/10.1109/90.811451

[3] Marbach, P. and Berry, R. (2002) Downlink Resource Allocation and Pricing for Wireless Networks. Proceedings of 21 st Annual Joint Conference of the IEEE Computer and Communications Societies, Vol. 3, New York, 23-27 June 2002, 1470-1479.

[4] Heikkinen, T. (2000) On the Quality of Service and Pricing in a Multiservice Network. 2000 IEEE International Conference on Multimedia and Expo, Vol. 3, New York, 30 July-2 August 2000, 1623-1626. https://doi.org/10.1109/ICME.2000.871081

[5] Faisal Iqbal, M., Zahid, M., Habib, D. and Kurian John, L. (2019) Efficient Prediction of Network Traffic for Real-Time Applications. Journal of Computer Networks and Communications, 2019, Article ID: 4067135.

https://doi.org/10.1155/2019/4067135

[6] Boutaba, R., Salahuddin, M.A, Limam, N., Ayoubi, S., Shahriar, N., Estrada-Solano, F. and Caicedo, O.M. (2018) A Comprehensive Survey on Machine Learning for Networking: Evolution, Applications and Research Opportunities. Journal of Internet Services and Applications, 9, Article No. 16. https://doi.org/10.1186/s13174-018-0087-2

[7] Ramakrishnan, N. and Soni, T. (2018) Network Traffic Prediction Using Recurrent Neural Networks. 2018 17th IEEE International Conference on Machine Learning and Applications, Orlando, 17-20 December 2018, 187-193.

https://doi.org/10.1109/ICMLA.2018.00035

[8] Chen, W.B., Shang, Z.H. and Chen, Y.H. (2019) A Novel Hybrid Network Traffic Prediction Approach Based on Support Vector Machines. Journal of Computer Networks and Communications, 2019, Article ID: 2182803. https://doi.org/10.1155/2019/2182803

[9] Nie, L.S., Jiang, D.D., Yu, S. and Song, H.B. (2017) Network Traffic Prediction Based on Deep Belief Network in Wireless Mesh Backbone Networks. 2017 IEEE Wireless Communications and Networking Conference, San Francisco, 19-22 March 2017, 1-5. https://doi.org/10.1109/WCNC.2017.7925498

[10] Abdel-Hadi, A. and Clancy, C. (2013) A Robust Optimal Rate Allocation Algorithm and Pricing Policy for Hybrid Traffic in 4G-LTE. 2013 IEEE 24th Annual Interna- 
tional Symposium on Personal, Indoor, and Mobile Radio Communications, London, 8-11 September 2013, 2185-2190.

https://doi.org/10.1109/PIMRC.2013.6666506

[11] Harks, T. (2004) Utility Proportional Fair Bandwidth Allocation: An Optimization-Oriented Approach. 2004 International Workshop on Quality of Service in Multiservice IP Networks, Catania, 2-4 February 2004, 61-74.

https://doi.org/10.1007/978-3-540-30573-6 5

[12] Tychogiorgos, G., Gkelias, A. and Leung, K.K. (2012) Utilityproportional Fairness in Wireless Networks. 2012 IEEE 23rd International Symposium on Personal, Indoor and Mobile Radio Communications, Sydney, 9-12 September 2012, 839-844. https://doi.org/10.1109/PIMRC.2012.6362900

[13] Gers, F.A, Schmidhuber, J. and Cummins, F. (1999) Learning to Forget: Continual Prediction with LSTM. 9 th International Conference on Artificial Neural Networks, Edinburgh, 7-10 September 1999, 850-855. https://doi.org/10.1049/cp:19991218

[14] Trinh, H.D., Giupponi, L. and Dini, P. (2018) Mobile Traffic Prediction from Raw Data Using LSTM Networks. 2018 IEEE 29th Annual International Symposium on Personal, Indoor and Mobile Radio Communications, Bologna, 9-12 September 2018, 1827-1832. https://doi.org/10.1109/PIMRC.2018.8581000

[15] Telecom Italia (2015) Telecommunications-SMS, Call, Internet-MI. Harvard Dataverse, Cambridge. https://doi.org/10.7910/DVN/EGZHFV 